\title{
Malignant rhabdoid tumor in the renal allograft of an adult transplant recipient: a unique case of a rare tumor
}

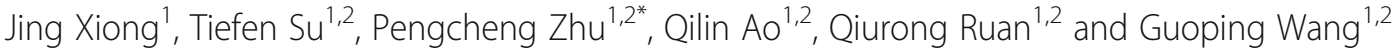

\begin{abstract}
Background: Renal transplant recipients have increased risk for developing malignant diseases because of immunosuppression or donor-to-recipient transmission. Malignant rhabdoid tumor (MRT) is a rare, highly aggressive and lethal tumor primarily affecting the kidney of infants and young children. MRT has not been reported in the renal allograft of an adult recipient after kidney transplantation.
\end{abstract}

Case presentation: In this report, a 47-year-old woman who received a kidney transplantation from an infant donor and developed a mass in the transplanted kidney is presented. Pathological examinations revealed a malignant tumor with rhabdoid cells morphologically and the loss of INI1 expression immunohistochemically. The diagnosis of malignant rhabdoid tumor in the transplanted kidney was made. We confirmed that donor-to-recipient malignancy transmission was the cause of MRT in the transplanted kidney by fluorescence in situ hybridization (FISH) and short tandem repeat (STR) analysis.

Conclusion: To our knowledge, this is the first case of MRT in an adult renal allograft recipient. This report highlights the importance of the criteria for selection of donors to screen possible malignant tumors transmission.

Keywords: Malignant rhabdoid tumor (MRT), SMARCB1/INI1, Kidney transplantation, Donor-to-recipient malignancy transmission

\section{Background}

Graft survival has improved considerably in kidney transplantation thanks to potent immunosuppression. On the other hand, the overall incidence of malignancy after renal transplantation is much higher than in the general population [1]. The major reasons for this increased risk are thought to be perturbation of immune surveillance mechanisms secondary to the chronic use of immunosuppressive agents and donor-to-recipient malignancy transmission [2]. Malignant rhabdoid tumor (MRT) of the kidney is a highly aggressive tumor of infancy and childhood [3]. This tumor is characterized by noncohesive tumor cells with eccentric nuclei and

\footnotetext{
* Correspondence: zhupengcheng@hust.edu.cn

${ }^{1}$ Institute of Pathology, Tongji Hospital, Tongji Medical College, Huazhong University of Science and Technology, 1095 Jiefang Avenue, Wuhan 430030, People's Republic of China

${ }^{2}$ Department of Pathology, School of Basic Medical Science, Tongji Medical College, Huazhong University of Science and Technology, Wuhan 430030, People's Republic of China
}

eosinophilic cytoplasm morphologically and deletion/ mutation of the SMARCB1/INI1 gene located on chromosome 22q 11.2 genetically [4]. Here, we report a rare case of MRT arising from a renal allograft in a 47year-old female patient who received a kidney transplantation for renal failure. We confirmed that the donor-to-recipient malignancy transmission was the cause of MRT in the transplanted kidney by fluorescence in situ hybridization (FISH) and short tandem repeat (STR) analysis. To our knowledge, this is the first case of MRT in an adult renal allograft recipient.

Approval was obtained from Ethics committee of Tongji Hospital, Tongji Medical College, Huazhong University of Science and Technology. Written informed consent was obtained from the patient in this study.

\section{Case presentation \\ Case report}

A female patient was diagnosed with thrombocytopenia due to persistent gum bleeding and lower extremity 
congestion at the age of 40 years in a local hospital. Subsequently, she was detected to have hypertension. She took nifedipine for hypertension for three years, but her health conditions worsened. A renal function examination showed an elevated serum creatinine (Scr) level $(342 \mu \mathrm{mol} / \mathrm{l})$, and symptomatic treatment did not improve renal function before dialysis. After six months of dialysis, the patient underwent kidney transplant operation since a 56-day-old infant who died from a central nerve system (CNS) tumor (suspected astrocytoma without pathological evidence) came to the hospital as a kidney donor by his parents and was found to be suitable for kidney donation. There was no history of rhabdoid tumors in the donor family history.

Dual kidneys were transplanted in the right iliac fossa of the recipient (Fig. 1a and b, black arrows), and the original kidneys were not removed (Fig. 1a, white arrows). Her immediate post-transplant situation was placid. Four months later, she developed hematuria and accelerated graft dysfunction. Ultrasound and computed tomography (CT) showed a 73-mm mass within the enlarged internal transplanted kidney (Fig. 1a and b, triangle), and the external transplanted kidney was unchanged. The enlarged transplanted kidney was, therefore, removed by surgery.
Follow-up revealed that the recipient was alive and well without recurrence 10 months after diagnosis, and the other intact transplanted kidney showed no mass by routine $\mathrm{CT}$ scan.

\section{Pathological findings}

The removed transplanted kidney measured $9.0 \times 7.9 \times$ $7.5 \mathrm{~cm}$. The cut surface showed a $7.4 \times 6.3 \times 6.0 \mathrm{~cm}$ mass without a capsule located in the upper pole, replacing almost the entire kidney (Fig. 1c). The samples were fixed in $4 \%$ formalin and embedded in paraffin. Sections were cut and stained with hematoxylin \& eosin (H\&E). For immunohistochemistry, the paraffin-embedded tissue blocks were sliced to 3-4 $\mu \mathrm{m}$ thickness. After deparaffinization, antigen retrieval with heat and 3\% hydrogen peroxide $\left(\mathrm{H}_{2} \mathrm{O}_{2}\right)$ methanol solution treatment was done for $30 \mathrm{~min}$ to eliminate nonspecific reaction with proteins. The primary antibodies applied are given in Table 1. Immunostaining was performed by an enhancement method based on repetitive microwave heating of slides that were placed into $0.01 \mathrm{M}$ citrate buffer at $\mathrm{pH}$ 6.0. Binding of primary antibodies was visualized with an Envision two-step method. Diaminobenzidine was used as chromogen, and nuclei were stained with Mayer's hematoxylin. Appropriate positive controls were included.

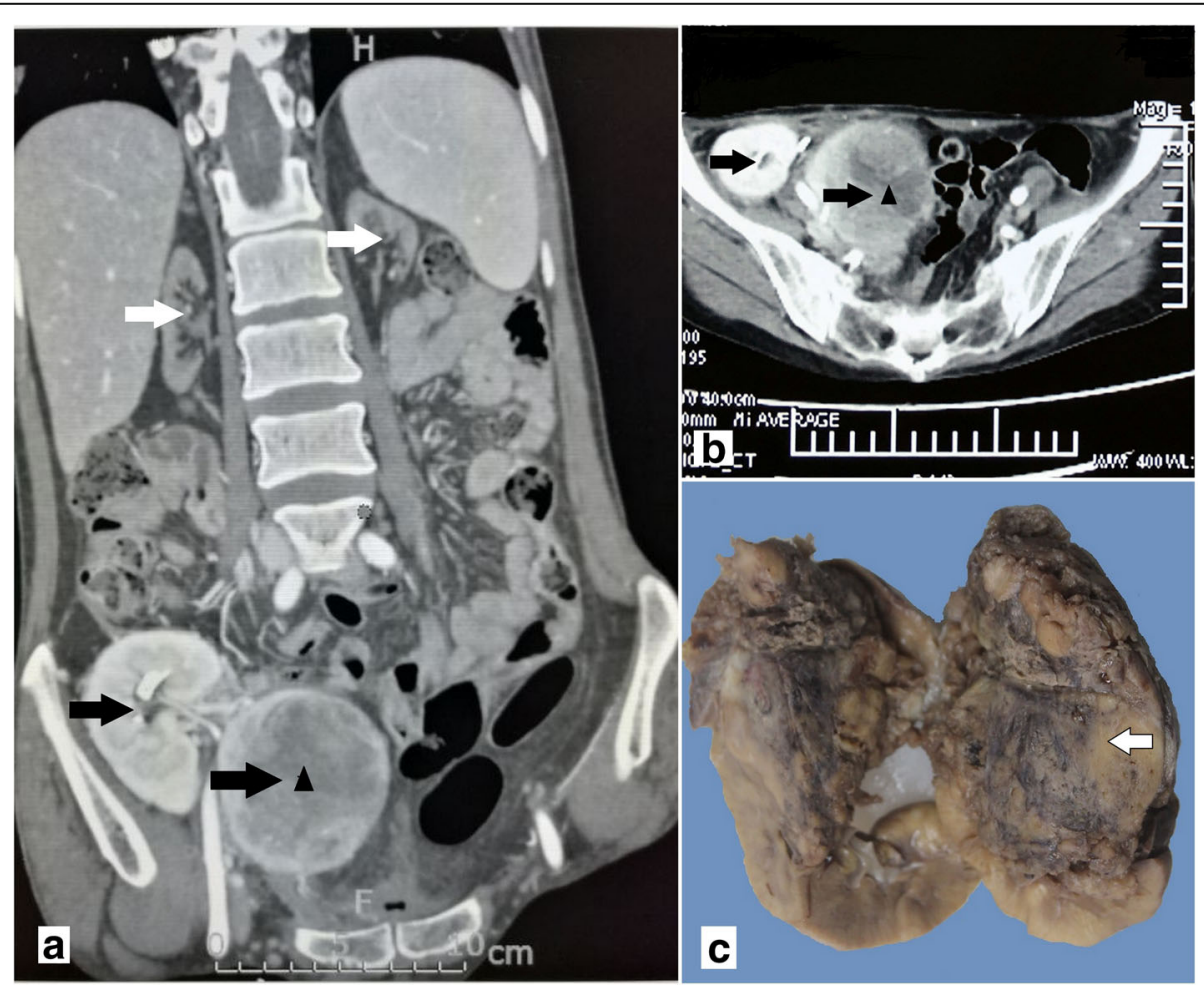

Fig. 1 Computed tomography (CT) scan revealed that both original kidneys exhibited atrophy (a, white arrows) and a large mass (a and $\mathbf{b}$, triangle) measuring $7.4 \times 6.3 \times 6.0 \mathrm{~cm}$ with a mixed density in the upper pole of the inner transplanted kidney (a and $\mathbf{b}$, black arrows). c. Macroscopic features of MRT of the transplanted kidney. The cut surface of the mass was white to grayish (arrow) 
Table 1 Antibodies and dilutions used in the evaluation of malignant rhabdoid tumor in transplanted kidney

\begin{tabular}{lll}
\hline Antibody & Dilution & Source \\
\hline Pancytokeratin & $1: 100$ & Dako \\
EMA & $1: 50$ & Dako \\
Vimentin & $1: 100$ & Novocastra \\
INI-1 & $1: 100$ & Dako \\
CD99 & $1: 50$ & Dako \\
WT-1 & $1: 100$ & Dako \\
Desmin & $1: 200$ & Dako \\
Myogenin & $1: 100$ & Dako \\
MyoD1 & $1: 100$ & Dako \\
CAIX & $1: 100$ & Santa Cruz \\
CD34 & $1: 100$ & Dako \\
CD56 & $1: 100$ & Novocastra \\
CgA & $1: 100$ & Dako \\
Syn & $1: 100$ & Dako \\
GATA-3 & 1:100 & Santa Cruz \\
CD10 & $1: 100$ & Novocastra \\
CD117 & $1: 200$ & Dako \\
CK5/6 & $1: 200$ & ZYMED \\
P63 & $1: 25$ & Novocastra \\
AMACR & $1: 100$ & Dako \\
TFE-3 & $1: 200$ & Santa Cruz \\
S-100 & $1: 1000$ & Dako \\
HMB45 & $1: 100$ & \\
MelanA & & Dako \\
CD38 & $1: 100$ & \\
CD138 & N167 & Nako \\
\hline
\end{tabular}

Microscopic examination showed patternless sheets or nests of noncohesive, uniform, round and oval tumor cells having eccentric nuclei with macro-nucleoli and abundant eosinophilic cytoplasm (Fig. 2a, b). Some of the neoplastic cells had round, eosinophilic, hyalinewhorled paranuclear cytoplasmic inclusions.

\section{Immunohistochemistry and molecular analysis}

Immunohistochemically, the tumor cells were diffusely positive for vimentin and CD99, focally positive for epithelial membrane antigen (EMA) and cytokeratin, but negative for SMARCB1/INI1(Fig. 2c). All other markers (WT-1, desmin, myogenin, myogenic differentiation 1(MyoD1), carbonic anhydrase IX(CAIX), CD34, CD56, chromogranin A (CgA), synaptophysin (Syn), GATA binding protein 3 (GATA-3), CD10, CK5/6, P63, CD117, P504s, transcription factor binding to IGHM enhancer 3 (TFE-3), S-100, HMB45, MelanA, CD38, and CD138) were not expressed in the tumor cells. The Ki67 labeling index was approximately $80 \%$ (Fig. 2d).

For FISH analysis of SMARCB1/INI1 and sex chromosomes, paraffin-embedded 5- $\mu \mathrm{m}$ sections were deparaffinized. A probe specific for SMARCB1/INI1 (Empire Genomics, NY, USA) and a dual-color interphase FISH probe set for the $\mathrm{X}$ centromere (CEP $\mathrm{X}$ ) and $\mathrm{Y}$ centromere (CEP Y) (GP Medical Technologies Inc., Beijing, China) were used to detect any abnormality of SMARCB1/INI1 and male (XY) donor or female (XX) recipient cells present in the kidney tumor specimen according to the manufacturers' protocols. The fluorescence signals were analyzed using an Olympus BX51 fluorescence microscope (Olympus, Tokyo, Japan) equipped with appropriate filters and imaged using Vysis software. At least 200 cells were scored.

FISH analysis showed that SMARCB1/INI1 was deleted (Fig. 3a), and most of the tumor cells had a male gonosomal complement with an $\mathrm{X}$ (green) and a $\mathrm{Y}$ (red) chromosome, which was consistent with donor origin (Fig. 3b).

For the short tandem repeat (STR)-based concordance study, genomic DNA was extracted from tumor tissue, normal renal tissue samples of the transplanted kidney and skeletal muscle of the recipient using the Chelex100 protocol and subsequently quantified with the Nanodrop 2000 spectrophotometer (Thermo Fisher Scientific, MA, USA). Multiplex genotyping was performed with the EX22 STR kit (AGCU ScienTech Incorporation, Wuxi, China) and the AmpFLSTR ${ }^{\odot}$ Identifiler $^{\odot}$ PCR Amplification kit (Thermo Fisher Scientific, MA, USA) on the case samples according to the manufacturers' recommendations. The experimental procedures followed internal laboratory control standards and kit controls.

The STR results showed that of the 22 microsatellite markers tested, differences were found between tumor cells (Fig. 4a) and skeletal muscle from the recipient (Fig. 4c) in all 22 informative alleles, and a Y chromosome was detected in the tumor cells. We concluded that the tumor was derived from the donor (Fig. 4b).

\section{Discussion}

Primary tumors in the kidney allograft recipients have often been reported since the advent of potent immunosuppression. The most common malignant tumors of organ transplant recipients are post-transplant lymphoproliferative diseases and skin cancer [5]. To our knowledge, this is the first case of MRT in a kidney transplanted into an adult.

MRT is a rare neoplasm that occurs mainly in the kidney in children less than 1 year of age, with an aggressive clinical course [6]. Some cases with histologic appearance similar to that arising in the kidney have been 


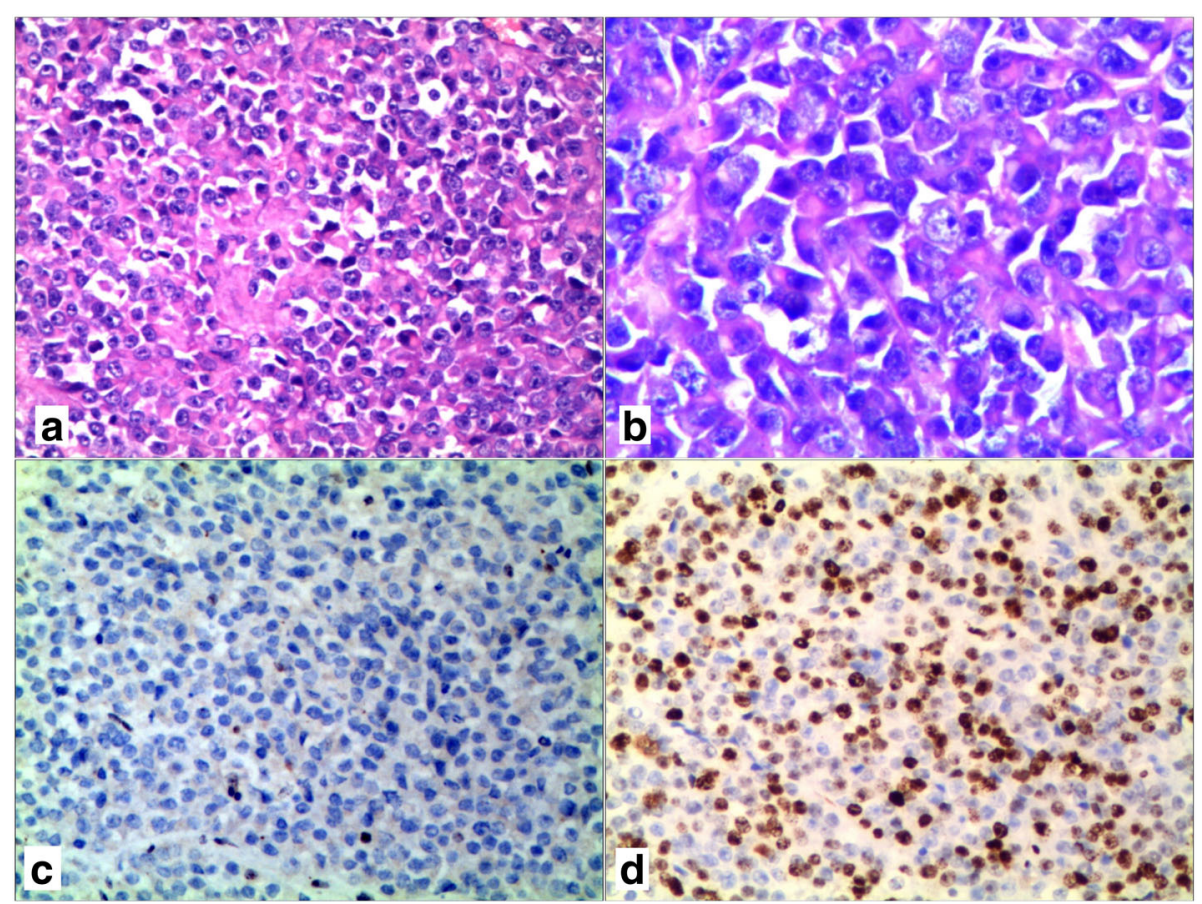

Fig. 2 The tumor cells showed patternless sheets or nests of noncohesive, uniform, round and oval tumor cells having eccentric nuclei with macro-nucleoli and abundant eosinophilic cytoplasm (a, H\&E $\times 200$ and b, H\&E $\times 400)$. c tumor cells showing loss of staining for INI1 immunohistochemically. d The Ki67 labeling index showed high proliferation rates of the tumor cells immunohistochemically

described in virtually every extrarenal anatomic site, including soft tissue, retroperitoneum, mediastinum, orbit, gastrointestinal system, uterus, and most prominently the CNS, where they are referred to as atypical teratoid/ rhabdoid tumors (AT/RT) [7]. As a distinct and unique type of malignant tumor, MRT has the characteristic appearance of patternless sheets of noncohesive cells with abundant cytoplasm and eosinophilic inclusions, as well as specific molecular aberrations involving SMARCB1 (hSNF5/INI1), which can be identified by a lack of staining with INI1 immunohistochemically. Fewer than 10 cases of MRTs are reported in adult patients in the English-language literature $[8,9]$, and only one case of MRT in the native kidney in an adult patient following kidney transplantation was reported [10]. In the present study, the mass in the transplanted kidney was diagnosed as MRT based on the histopathological features and immunohistochemical findings.

Other primary renal neoplasms and certain epithelioid or rhabdoid cell lesions arising from the kidney and
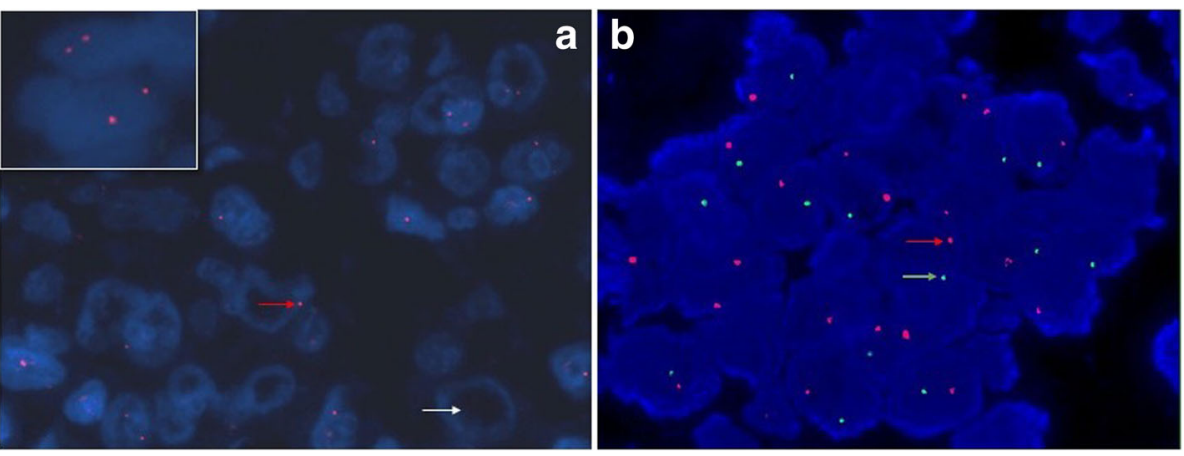

Fig. 3 Fluorescence in situ hybridization (FISH) analysis: a Deletion of SMARCB1/INI1 is evidenced by loss of one (red arrow) or both (white arrow) probe signals (red) in tumor nuclei, while two copies are retained in the nuclei of normal renal tubular epithelial cells (insert). $\mathbf{b}$ FISH analysis of sex chromosomes showed tumor cells had a male gonosomal complement (positive results for the $\mathrm{X}$ (green arrow) and $\mathrm{Y}$ chromosomes (red arrow), consistent with donor origin) 


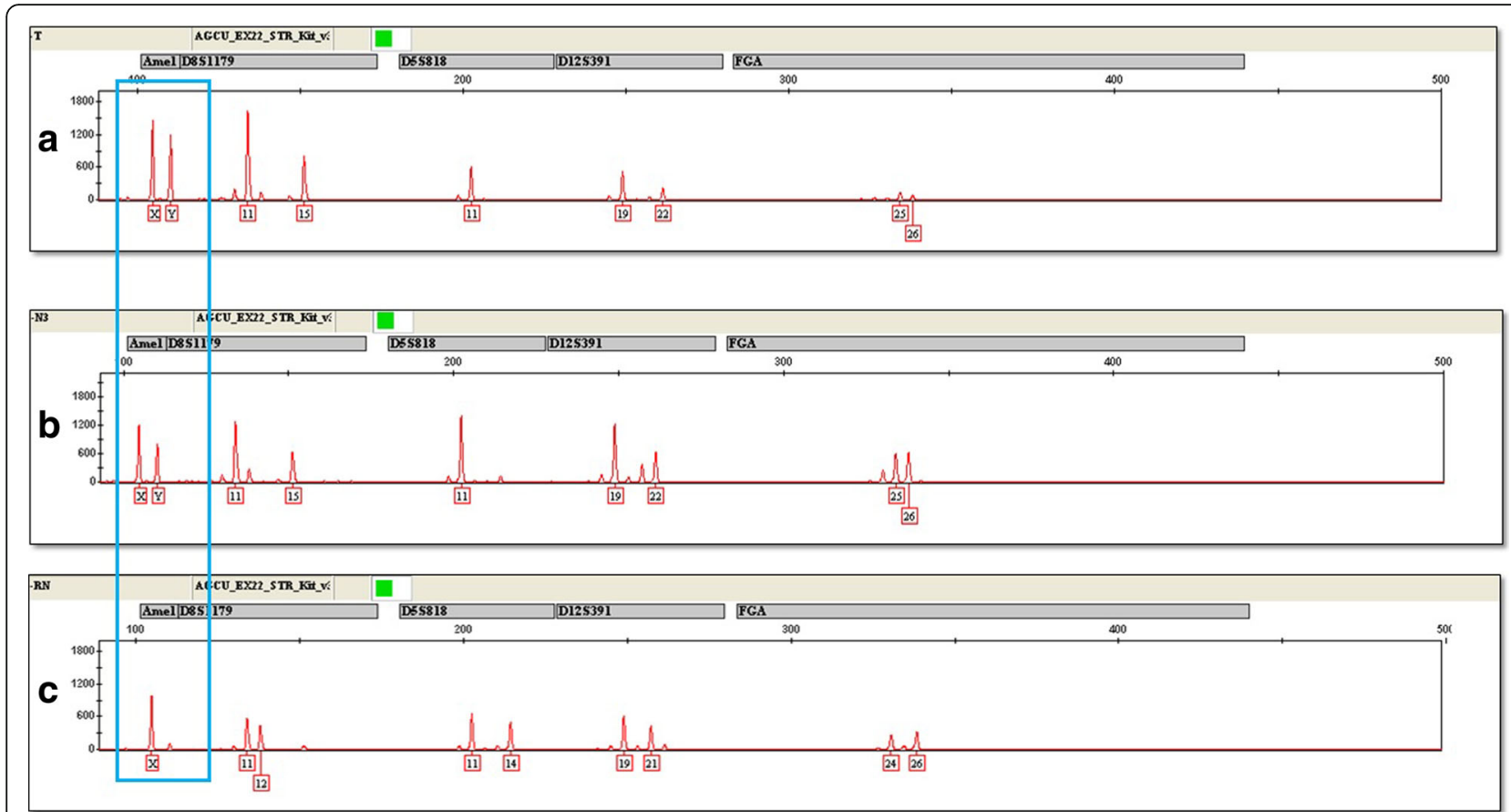

Fig. 4 Short tandem repeat (STR)-based concordance study of the 22 microsatellite markers tested, differences were found between tumor (a) and skeletal muscle from the recipient (c) in all 22 informative alleles, and a Y chromosome (blue box) was detected, indicating that the MRT was derived from the donor cells (b)

urinary tract should be distinguished from MRT upon diagnosis. Nephroblastoma (Wilms tumor), a common solid tumor of childhood, typically shows a distinct triphasic pattern with blastema, stromal, and epithelial components and without INI1 deficiency. Epithelioid sarcoma is a malignant tumor affecting an older age group than MRT, with prominent rhabdoid cells and loss of nuclear INI1 in most cases, and it often shows CD34positive staining, mainly located in the extremities. Other malignancies, including rhabdomyosarcoma, myoepithelioma in soft tissue, epithelioid angiosarcoma, and epithelioid malignant peripheral nerve sheath tumor (MPNST), can be distinguished from MRT by their specific markers and INI1 expression. Loss of INI1 is also helpful to differentiate MRT from other primary renal neoplasms with rhabdoid differentiation, including clear cell renal carcinoma, chromophobe cell renal carcinoma, and other malignances in the urinary system $[11,12]$.

Due to the rarity of MRT in transplanted kidney, FISH and STR analysis were used to determine the tumor origin in this case. The results showed a Y chromosome in the tumor cells, which was consistent with donor origin. In general, malignant tumors in allografts can develop in two ways: de novo development in the allograft of an immunosuppressed recipient [1] and donor-to-recipient malignancy transmission $[13,14]$. In the present case, the recipient's medical record revealed that the deceased donor was a 56-day-old infant who died of a CNS tumor (suspected astrocytoma without pathology or autopsy evidence), and there was no evidence of a mass in the allograft by imaging examination before transplantation.

There was no history of rhabdoid tumors in the donor family history.

Although we cannot refute the diagnosis of astrocytoma and prove the presence of AT/RT, we strongly suspect the CNS tumor of the infant donor was an AT/RT, a tumor that predominantly affects infants and young children and has histological features similar to MRT in kidney, with poor prognosis. FISH and STR analysis of the recipient's tumor was consistent with donor origin. Therefore, we consider donor-to-recipient malignancy transmission likely in this case, making this the first report of MRT in a renal allograft of an adult recipient following renal transplantation.

Donor-to-recipient malignancy transmission could occur in two ways. First, there could be some metastatic cells in the transplanted kidney that come from a primary tumor in another organ and then transfer in the organ recipient. Second, post-transplant lymphoproliferative disorder, the most common post-transplant malignancy, could come from malignant change of passenger leukocytes in the transplanted organ. We favor the former explanation in this case, as it was likely that the tumor cells from AT/RT in the CNS of the pediatric donor metastasized to the kidney, which was transplanted to the adult female recipient. 
According to strategy and rules for organ screening and acknowledgment before transplantation, donors with a history of lung cancers, sarcomas and grade IV CNS neoplasms are considered unacceptable, but donors with a past with grade I-II CNS tumors are acceptable. In the present case, the donor, a 56-day-old infant who died of a CNS tumor with a suspected diagnosis of astrocytoma, was not reasonable because AT/RT should have been considered in the differential diagnosis before the transplantation.

Since the number of patients requiring transplantation therapy is growing, there is increasing demand for donor organs. The expansion of the general donor pool has led to the inclusion of donors of extreme ages and donors who may possibly transmit disorder to the recipients. In any case, the peripheral donors' organ utility is frequently connected with a more serious danger of undiagnosed disease including malignant tumors and some infections [15]. Thus, the evaluation of infants or very young children with CNS tumors as donor candidates for kidney transplantation should be investigated for the synchronous presence of kidney tumors.

\section{Conclusion}

We report a unique case of MRT in an adult renal allograft recipient after kidney transplantation with characteristic histologic features and loss of INI1 expression. This report highlights the importance of the criteria for selection of donors to screen possible malignant tumors transmission in living and dead donor transplants.

\section{Abbreviations \\ AT/RT: Atypical teratoid/rhabdoid tumor; CAIX: Carbonic anhydrase IX; CEP $X: X$ centromere; CEP Y: Y centromere; CgA: Chromogranin A; CNS: Central nervous system; CT: Computed tomography; EMA: Epithelial membrane antigen; FISH: Fluorescence in situ hybridization; GATA-3: GATA binding protein 3; H\&E: Hematoxylin \& eosin; INI1: Integrase interactor 1; MPNST: Malignant peripheral nerve sheath tumor; MRT: Malignant rhabdoid tumor; MyoD1: Myogenic differentiation 1; SMARCB1: SWI/SNF-related matrix- associated actin-dependent regulator of chromatin subfamily B member 1; STR: Short tandem repeat; Syn: Synaptophysin; TFE3: Transcription factor binding to IGHM enhancer 3}

\section{Acknowledgements}

The authors thank Dr. Qinlu Li from the Department of Hematology, Tongji Hospital, for her help in sex chromosome FISH analysis. We also thank Dr. Chao Xiao from the Department of Forensic Medicine, Tongji Medical College, for his help in STR analysis.

\section{Funding}

This work was supported by a research grant (81502296 to XJ) from the National Natural Science Foundation of China.

Availability of data and materials

Please contact the author directly for data requests.

\section{Authors' contributions}

XJ, STF and ZPC completed the clinical data collection, analyzed part of the morphology studies, participated in the study design and drafted the manuscript. XJ and STF performed the morphology, immunohistochemistry and molecular studies. XJ and STF interpreted the clinical data. AQL, RQR and WGP conceived of the study, participated in its design and coordination and helped draft the manuscript. All the authors read and approved the final manuscript.

\section{Ethics approval and consent to participate}

This study was approved by the Ethics committee of Tongji Hospital, Tongji Medical College, Huazhong University of Science and Technology.

\section{Consent for publication}

Written informed consent for publication of the clinical details and/or clinical images was obtained from a relative of the patient. A copy of the consent form is available for review by the Editor of this journal.

\section{Competing interests}

The authors declare that they have no competing interests.

\section{Publisher's Note}

Springer Nature remains neutral with regard to jurisdictional claims in published maps and institutional affiliations.

Received: 27 June 2017 Accepted: 8 December 2017

Published online: 19 December 2017

\section{References}

1. Doycheva I, Amer S, Watt KD. De novo malignancies after transplantation: risk and surveillance strategies. Med Clin North Am. 2016;100:551-67.

2. Desai R, Collett D, Watson CJ, Johnson P, Evans T, Neuberger J. Estimated risk of cancer transmission from organ donor to graft recipient in a national transplantation registry. Br J Surg. 2014;101:768-74.

3. Weeks DA, Beckwith JB, Mierau GW, Luckey DW. Rhabdoid tumor of kidney. A report of 111 cases from the National Wilms' tumor study pathology center. Am J Surg Pathol. 1989;13:439-58.

4. Hollmann TJ, Hornick JL. INI1-deficient tumors: diagnostic features and molecular genetics. Am J Surg Pathol. 2011;35:e47-63.

5. Saleeb R, Faragalla H, Yousef GM, Stewart R, Streutker CJ. Malignancies in a renal transplant population: the St. Michael's hospital experience. Urol Ann. 2016;8:163-7.

6. Schmidt D, Harms D, Zieger G. Malignant rhabdoid tumor of the kidney. Histopathology, ultrastructure and comments on differential diagnosis. Virchows Arch A Pathol Anat Histopathol. 1982;398:101-8.

7. Torchia J, Picard D, Lafay-Cousin L, Hawkins CE, Kim SK, Letourneau L, et al. Molecular subgroups of atypical teratoid rhabdoid tumours in children: an integrated genomic and clinicopathological analysis. Lancet Oncol. 2015;16: 569-82.

8. Podduturi V, Campa-Thompson MM, Zhou XJ, Guileyardo JM. Malignant rhabdoid tumor of the kidney arising in an adult patient. Proc (Bayl Univ Med Cent). 2014;27:239-41.

9. Peng HQ, Stanek AE, Teichberg S, Shepard B, Kahn E. Malignant rhabdoid tumor of the kidney in an adult: a case report and review of the literature. Arch Pathol Lab Med. 2003;127:e371-3.

10. Sato Y, lizuka J, Imai K, Sawada Y, Komatsu T, Yago R, et al. Case report of rhabdoid tumor of the kidney occurring in own kidney following kidney transplantation from the living relative. Nihon Hinyokika Gakkai Zasshi. 2010; 101:683-8.

11. Przybycin CG, McKenney JK, Reynolds JP, Campbell S, Zhou M, Karafa MT, Magi-Galluzzi C. Rhabdoid differentiation is associated with aggressive behavior in renal cell carcinoma: a clinicopathologic analysis of 76 cases with clinical follow-up. Am J Surg Pathol. 2014;38:1260-5.

12. Shannon BA, Cohen RJ. Rhabdoid differentiation of chromophobe renal cell carcinoma. Pathology. 2003;35:228-30.

13. Xiao D, Craig JC, Chapman JR, Dominguez-Gil B, Tong A, Wong G. Donor cancer transmission in kidney transplantation: a systematic review. Am J Transplant. 2013;13:2645-52.

14. Green M, Covington S, Taranto S, Wolfe C, Bell W, Biggins SW, et al. Donorderived transmission events in 2013: a report of the organ procurement transplant network ad hoc disease transmission advisory committee. Transplantation. 2015;99:282-7.

15. Bozkurt B, Kilic M. Marginal donors in renal transplantation. Transplant Proc. 2015;47:1273-81. 